\title{
TRAVAUX DE LA STATION DE RECHERCHES APICOLES DE BURES SUR YVETTE EN 1958
}

PAR

\author{
Rémy CHA UVIN
}

\section{Pollen et golép royale.}

I,es phénomènes de vieillissement qui conduisent à une lente dégradation des propriétés hyperglycémiantes de la gelée royale se rencontrent aussi dans le cas du pollen, comme le signale une note de Chacvin et I,AVIF. Ici, les auteurs ne s'adressent plus aux propriétés hyperglycémiantes des produits, mais à leur activité antibiotique (voir Chauvin et IAviE, Ann. Inst. Pasteur, 90, I956, 523). Cette activité est faible dans le pollen fraîchement récolté sur la plante ou qui vient de tomber dans le tiroir de la trappe à pollen; mais si le même pollen est stocké pendant huit jours par les abeilles dans leurs alvéoles, il contient trois fois plus d'unités antibiotiques; l'augmentation continue, en se ralentissant toutefois, au cours du stockage (ajoutons que le pouvoir hyperglycémiant du pollen apparaît, puis se développe d'une manière analogue pendant le stockage). I, 'action antibiotique de la gelée varie aussi beaucoup et se dégrade rapidement à la température ordinaire, plus lentement à la glacière.

\section{Déterminisme de la construction des cellules royales.}

Continuant ses travaux sur la construction des cellules royales, VUII,AUME distingue les limites d'application des théories de BUTI,ER sur l'ectohormone des reines d'abeille, qui arrête dans les ruches la construction des cellules royales. Flle n'inhibe en réalité que les phases initiales de la construction spontanée des cellules, quand on se borne à enlever la reine dans la ruche; alors, si on réintroduit la reine au bout de peu de temps, ou bien la construction n'est pas entreprise, ou bien les ébauches de cellules royales sont détruites. Mais lorsque l'expérimentateur introduit dans une colonie, même non orpheline, des cupules contenant déjà 
une jeune larve, l'élevage est entrepris sans difficultés, mêne parfois si la reine est libre ; il suffit en tont cas de l'empêcher de venir à proximité immédiate des cellules (par une grille à reine) pour que l'expérience réussisse.

Ces possibilités d'adaptation des abeilles montront néannoins, comme il fallait $s$ attendre, cettaines limites ; par exemple, Vumatrate a tenté de laire élever plusicurs larves dans tule seule celluk royale de grandes dimensions, cloisonnée on non; mais les ouvrières rejettent aussitôt les larves ainsi introduites. I a raison en est sans doute que des cellules royales mêne soudées à la base comme il arrive fréquemment, ont toujours des bords libres; alors que dans la grande cupule employée dans les expériences de Vínuatme, les bords sont confondus. P'ar contre, le groupement des larves par deux daus les cupules ordinaires rencontre parfois un certain succès et aboutit à des cellules operculées à double tête; toutefois, ces cellules ne renferment à la fin qu'une seule larve en voie de nymphose, et l'autre partie de la cellule est vide.

Ians un autre travail, Vur tamp, examine le rôle de la propolis dans l'inhibition de la construction dess cellules royales. Il avait déjà signalé le fait que le simple badigeomnage des cupules de cire avec de la propolis empêche les abeilles de les transformer en cellules royales: I1 note maintenant que la substance inhibitrice peut être entraînée par la vapeur d'eau après saponification du produit; et qu'elle se rencontre également dans le bourgeon de peuplier et dans son essence; rappellons que d'après beaucoup d'auteurs la propolis est récoltée sur les bourgeons de peuplier. I,essence de propolis ou de bourgeons de penplier inhibe également l'étirage des cires gaufrées ; il s'agit donc de substances inhibitrices de la construction en général. Cependant, l'ectohornone des reines, appliquée en badigeonnage, n'arrête que le remaniement et la transformation en cellules royales; mais non pas l'étirage des cellules d'ouvrières: Sa spécificité est donc plus grande que celle de la propolis.

\section{Ia construction des rayons.}

Lile a fait l'objet d'une communication spéciale au Congrès International d'Entomologie de Montréal, oì DARchen montre l'influence de la grappe cirière sur le développement du rayon ; en pratiquant 1'ablation de diverses portions du bord du rayon, il constate que les abeilles le réparent en rétablissant la forme normale. Des obstacles insérés à différents niveaux provoquent des perturbations différentes : près de la zone d'insertion du rayon sur le cadre, elles sont peu marquées; nais auprès du sommet, elles ont pour conséquence une hernie de la cire de part et d'autre de l'obstacle. Une toile métallique reconvrant une face interrompt complètement la construction dans la zone intéressée, spécialement 
si la toile se trouve dans la zone sensible du sommet du rayon. L'insertion de feuilles métalliques sur la tranche du rayon arrête complètement la construction, si leur largeur dépasse ro mm. Au-dessus de ce chiffre, il est nécessaire, pour rétablir la construction, de percer dans le métal des fenêtres d'une largeur déterminée. Toutes ces expériences manifestent l'existence d'une "infornation " circulant par des voies encore inconnules à travers les abeilles réunies en chaîne cirière. Dans une note plus récente (I958) DARCHEN signale que les abeilles peuvent tordre de manière à la rendre parallèle, une lame de cire gaufrée insérée entre deux rayons perpendiculairement à leur plan. L,e comportement d'étirage peut être dissocié du comportement de torsion grâce à l'emploi de la propolis, qui permet la torsion, mais inhibe la construction.

\section{La construetion des cellules de mâles.}

A été étudiée de nouveau par IMrchex et Vuillaume. I)ans les abeilles formées en essaim, la reine inhibe la formation des cellules de mâles ; mais la présence de couvain sous forme de jeunes abeilles en train de naître arrête la construction des cellules d'ouvrières. I)ans le premier cas, 1'ectohormone de la reine serait peut-être responsable du phénomène ; dans le second, il conviendrait peut-être de penser à la fourniture par le couvain d'une substance inhibitrice de la construction des cellules d'ouvrières.

\section{Is butillage.}

Il a été étudié par I,HCOMTr et CocrTors à l'aide de l'or radioactif qui constitue un produit très acceptable pour le marquage des butineuses ; il a déjà permis de mettre en évidence l'hétérogénéité frappante de la dispersion des abeiles : deux champs situés côte à côte penvent être l'un visité, l'autre entièrement lélaissé, bien qu'ils contiennent l'un et l'autre des plantes mellifères. Sans doute doit-on faire intervenir ici toute une série de facteurs d'orientation complexes, les uns signalés par I orveiarx et les autres par I,ECOMTE.

La thèse de Iotveatx sur la récolte du pollen constitue un ouvrage trop considérable (206 pages) pour qu'on puisse le résumer facilement. Fxtrayons-en seulement deux conclusions particulièrement importantes et originales : l'auteur met d'abord en évidence, à l'aide d'une ingéniense technique, des phénomènes "d'acclimatation " déjà soupçonnés dans la pratique apicole. I)es groupes de ruches, transférées dans un environnement étranger, gardent des particularités de butinage fui les differencient nettement d'autres groupes ventus d'une antre zone, puis translérés dans ce même environnement. Dans un autre chapitre, Iouvkstrx met en évidence les variations progressives du taux d'azote des pollens récol- 
tés dans la ruche, ce qui doit certainement influencer la marche du développement à mesure que s'avance la saison; peut-être est-ce là un des facteurs responsables de l'acclimatation.

\section{Technologie du miel.}

I,OUVkAdX et 'lRUbert ont mesuré avec précision le processus d'échauffement d'un miel au cours de la fonte, et indiquent à cette occasion les précautions à prendre pour éviter les " points chauds " qui par surchauffes locales, abîment gravement la masse du miel.

BORNFCK, I,OUIS, I,OUVEAUX ont fait une enquête sur le marché du miel à Paris pendant 1'hiver I957-58. Cent points de vente ont été choisis au hasard sur toute la superficie de l'agglomération parisienne. Les enquêteurs ont attribués des notes à la présentation, au goùt à l'état de conservation du miel. I,eurs conclusions sont assez pessimistes : un trop grand nombre de miels sont mal préparés, parfois en pleine fermentation. L'origine indiquée est souvent fantaisiste, et l'on trouve sans peine des miels de romarin qui renferment une très forte proportion de miel d'acacia, ou des miels type Gâtinais, qui viennent du Mexique, etc. Un très gros effort reste à faire pour l'éducation des praticiens en ce qui concerne la techmologie du miel.

\section{TECHNIQUES APICOLES}

DARCuñ et IAVI: ont présenté aux abeilles divers échantillons de cires chimiques, extraites des produits pétroliers, et qui sont mélangés depuis de nombreuses années à la cire d'abeille, le plus souvent sans que l'apiculteur qui achète des cires gatıfrées en ait connaissance. Il semble d'après les critères adoptés par I)ARCHEN et IAvik, que la régularité des constructions n'est pas très bome si une certaine proportion de cire d'abeille n'est pas mélangée à la cire chimique; mais dans l'ensemble la plupart des cires chimiques sont bien acceptées et il ne semble pas que l'on puisse condamner leur mélange avec la cire d'abeille.

A propos de l'élevage des reines, Vuil,aume discute les différentes techniques d'orphelinage et de présentation des cellules royales artificielles employées par les apiculteurs. L'auteur les a d'abord perfectionnées en pratiquant la technique dite du starter permanent, où le paquet d'abeilles orphelin qui reçoit les cupules munies d'une larve est maintenu sans cesse en état de fonctionner par l'introduction réitérée de cadres de jeune convain. Mais l'on pent suppriner totalement l'emploi du starter et introduire directement les cupules dans le finisseur, si l'on a pris soin de greffer les jeunes larves sur une quantité plus importante de gelée royale (pure ou diluée). Il est vrai que cette technique ne réussit bien que dans un " finisseur " de grande taille (à I 7 cadres). Mais il est possible de simplifier 
beaucoup les opérations en conservant la reine à l'intérieur de la ruche, emprisonnée dans une cagette pour expédition de reine, comme en utilisent d'habitude les éleveurs. Pour amorcer l'élevage royal artificiel, la présence de la reine n'est pas un obstacle insurmontable, pourvu que ses mouvements soient restreints par l'emploi d'une petite cagette.

\section{Revue !̣énérales.}

Deux revues ont été publiées dans les Annales de l'Abeille, l'une par Chauvin sur divers points de la biologie des abeilles, l'autre par I,ECOMTE, sur le comportement des faux-bourdons.

En I957, à l'occasion du III Congrès de l'Union pour l'Etude des Insectes Sociaux, s'est tenu à la nouvelle Station expérimentale d'Apiculture de Montfavet, annexe de Bures-sur-Yvette une réunion du groupe de travail "Analyse pollinique ". Un fascicule spécial des Annales de l'Abeille y a été consacré. Ont pris la parole M. BARBIER (examen pollinique de quelques miels unifloraux), le $\mathrm{D}^{\mathbf{r}}$ Evensus (Pollenanalyse und Begutachtung von sedimentreichen Honigen) Louveaux (Recherches sur l'origine dans les miels du pollen de plantes entomophiles dépourvues

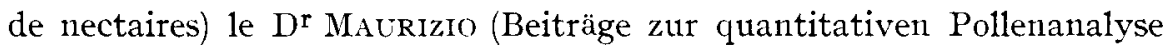
des Honigs).

\section{I,A STATION FXPÉRIMEN'TAIE, D'APICUL'TURE: DF: MONTFAVE'T}

Cette Station annexe fonctionne maintenant avec un équipement qui se complète peu à peu, sous la direction de Pierre I,Avik. Nous y avons constitué un département du miel avec une miellerie modèle (extracteur, maturateurs chauffés, pasteurisateur, chambre chaude conditionnée pour enlever du miel l'eau en excès, chambre froide pour la recristallisation du miel, etc.). I, rucher n'a pas encore atteint son plein développement (500 ruches) à cause des retards dus à une mauvaise année et à une crue subite qui a emporté 50 ruches. Nous pensons y conduire des recherches sur le miel et sur les races d'abeille, la sélection et l'entretien du rucher; alors que BuREs se confinera dans des recherches plus théoriques sur la biologie de l'abeille, son comportement, la technologie et la biochimie des produits de la ruche autres que le miel.

\section{REFITRLNCIN BIBLIOCRALHIQUTS}

(1) Chauvin (R.).-- 'Travaux de la station de Recherches Apicoles de Bures sur Yvette en $1957 A n n . A B$., I, I07-I I

(2) Chauvin (R.). - Biologie de l'abeille. Revte générale jusqu'en $195^{8}$. Ann. Ab., I, 4I-6)7. 
(3) Chauvin (R.) et Lavie (P.). - Le vieillissement du pollen emmagasiné par les abeilles et de la gelée rovale. C. R. Acad. Sc., 247, 2040-2.

(4) Courtois (G.) et Licom'te (J.). - Sur un procédé de marquage des abeilles butineuses au moyen d'un radioisotope. C. R. Acad. Sc., 247 , I $47-9$.

(5) DARChex (R.). - I es constructions sociales chez Apis mellifica. Travaud Cong. Int. Entom. Montréal, 529-36.

(6) IDARchen (R.). - I es Abeilles peuvent tordre une lame de cire pour la rendre parallèle aux rayons. C. R. Acad. Sc., 247, 2208-Io.

(7) Darchen (R.) Lavie (P.). - - Itude préliminaire de quelques qualités des cires gaufrées présentées aux abeilles $A n n . A B . \mathrm{r}, 7^{-9}$.

(i) J)ARChEx (R.) et Vuiliaume (M.). - I éterminisme de la construction des cellules de mâles et des cellules d'ouvrières chez Apis mellifica. Ann. Ab., I, 7-9.

(9) Lacome (J.). - Que savons-nous du comportement des faux-bourdons $f$ Ann. Ab., I, 3I-9.

(Io) Louveaux (J.). - Recherches sur l'origine dans le miel du pollen de plantes entomophiles dépourvues de nectaires. $A n n$. $A b$., I, 80-92.

(II) Louveaux (J.). - Recherches sur la récolte du pollen par les abeilles Apis mellifica L. Ann. Ab., I-II, $206 \mathrm{p}$.

(i2) Louveaux (J.), Truber'T (E.). - - Etude technique sur la fonte du miel cristallisé. $A n n . A b ., \mathrm{I}, \mathrm{I})-30$.

(13) Borneck (R.), Louis (J.), Louveaux (J.) - - Enquête sur le marché du miel à Paris pendant l'hiver I957-8. Ann. Abeille, I, I958, 223-46.

(I4) Vtritatim: (M.). I a théorie de Butler sur l'ectohormone des abeilles et ses limites. (. R. Acad. Sic, 247, 1927-().

(15) Vividaumi: (M.) Technique d'élevage des reines I. I e premier stade : élevage de cellules royales. Iitude critique. Ann. $A B$. I, I 89 -Ig6

(I6) Vundatm: (M.). - - Les substances inhibitrices de la construction des cellules royales che\% les abeilles. Ibid., 246, 1298-9.

(I7) Vuillatimi: (M.) Essai de modification des réactions instinctives des abeilles au cours de l'élevage royal. C.R. Aad.Sc., 2, 69-7I. 